\title{
WHO Response to COVID-19 and Technical Support in Uzbekistan: Capacity-building Focus
}

\section{Nazokat Kasymova}

\author{
WHO Country Office, Uzbekistan.
}

\begin{abstract}
The main aim of the present paper is to summarize the WHO efforts on capacity building (or capacity development of health care specialists and medical institutions to improve and retain the skills, knowledge, tools and competencies) both globally and at the national level in Uzbekistan. The joint approach of national governments, international organizations, health care providers and civil society is necessary to assure the effectiveness and quality of the response to the COVID-19 epidemic.
\end{abstract}

Keywords: WHO- Capacity building- Education/training- Uzbekistan

Asian Pac Environ Cancer, 3 (Suppl 1), 25-26

The health workforce crisis has disastrous implications for the health and well-being of millions of people, an increased lack of access to quality health services, in large part because of an imbalanced skill mix, and an uneven geographical distribution of health workers. The pool of health professionals is insufficient to meet the needs of the people, regardless of country or region [1].

Scaling up educational programs to produce multi-disciplinary service delivery teams - which include a carefully balanced mix of clinicians, community health workers and health managers - is clearly urgent and essential. However, simply increasing the numbers of workers will not be enough. The shortage of health workers is compounded by the fact that their skills, competencies, and clinical experience do not meet the needs or expectations of the population. WHO is working with a wide range of stakeholders to find answers for scaling up the health workforce to increase the quantity, and to improve the quality and relevance of health workers to meet the needs of the COVID-19 challenges and the $21^{\text {st }}$ century and contribute to better population health outcomes.

The WHO provides wide recourses for the use of healthcare workers: its strategies, plans, operations; technical guidelines; research; situation reports and media resources.

New and regularly updated technical guidelines
Submission Date: 06/15/2020Ａcceptance Date: 07/07/2020

related to COVID-19 on the WHO website cover a wide range of topics including critical preparedness, readiness and response actions for COVID-19, surveillance and case definitions, laboratory testing for 2019-nCoV in humans, country readiness, clinical care/patient management, infection prevention and control / water, sanitation and hygiene in health care facilities (WASH); four early investigations protocols that focused on transmission patterns, severity, clinical features and risk factors for infection (rebranded the WHO Unity Studies), essential resource planning, guidance for schools, workplaces $\&$ institutions, risk communication and community engagement, points of entry and mass gatherings, humanitarian operations, camps, and other fragile settings as well as refugees and migrants in non-humanitarian and non-camp settings, health workers, maintaining essential health services and systems [2].

The WHO proposes a new interactive, web-based, knowledge-transfer platform offering online courses to improve the response to health emergencies - "OpenWHO" or Global Specialist Training Courses. It enables the Organization and its key partners to transfer life-saving knowledge to large numbers of frontline responders [3]. Real-time training during global emergencies is critical for effective preparedness and response. Some courses are available in Russian such as Severe Acute Respiratory Infection (SARI) Treatment Facility Design; COVID-19:

\footnotetext{
Corresponding Author:

Dr. Nazokat Kasymova

WHO Country Office, Uzbekistan.

Email: kasymovan@who.int
} 
Operational Planning Guidelines and COVID-19 Partners Platform to support country preparedness and response; Infection Prevention and Control (IPC) for Novel Coronavirus (COVID-19); ePROTECT Respiratory Infections; Clinical Care Severe Acute Respiratory Infection; Emerging respiratory viruses, including COVID-19: methods for detection, prevention, response and control. The WHO Country Office (CO) in Uzbekistan shares all the presentations of these courses and has translated these materials into Uzbek. The COVID-19 channel provides knowledge and training for health professionals, decision-makers and the public regarding the outbreak of coronavirus disease but also can be used for self-education, better understanding of situations and as a resource.

The WHO in Uzbekistan also provided support for the development of National COVID-19 Guidelines, developed on the basis of WHO recommendations, which include a case management algorithm with questionnaires and assessment tools, infection prevention and control (IPC) issues, laboratory diagnostics, and clinical triage and patient management tactics, including the basics of managing severe and critical cases; management of children with COVID 19 and COVID-19 in pregnancy, childbirth and postpartum care. The National Guidelines are approved and available in Uzbek, Russian and partly in Karakalpak.

At present, the WHO CO team, together with local specialists, have developed training presentation materials for the training of healthcare workers based on the COVID-19 National Guidelines in the following areas: the algorithm for managing contact, suspected and confirmed cases of COVID-19; laboratory diagnostics and sampling for COVID-19 tests; organization of health facilities and the basics of the IPC; basics of home care and self-isolation; patient information, stress management; IPC modules in Russian and Uzbek. Training packages on the COVID-19 management of severe and critical cases in the context of Uzbekistan, materials on the characteristics of infection management in children, pregnant women, women in childbirth, oxygen therapy in maternity wards, management characteristics of patients with COVID-19 and NCDs are also being prepared.

For the general convenience WHO CO has created a common resource - the "COVID-19 training" shared folder, which contains all of the mentioned WHO training materials, including presentations of the official WHO Global Courses, the National Guidelines, brief WHO guidelines on various COVID-19 issues [4].

The Capacity Building Task Force with representatives of all national pre- and post-diploma medical institutions, main health workers and specialists, international organisations led by the Ministry of Health and WHO $\mathrm{CO}$ is actively working to response effectively to the COVID-19 in the country. According to clear message of Dr Tedros Adhanom Ghebreyesus, WHO Director-General the organization "will continue to provide technical, operational and logistics support to countries, and will continue to update and adapt our guidance according to local needs." [5].

\section{References}

1. Seifman Richard Global Health Workforce Crisis: What To Do. In Health, SDG Series, Society, United Nations July 11, 2019.// https://impakter.com/global-health-workforcecrisis-what/..

2. COVID-19: Technical Guidelines - https://www.who.int/ emergencies/diseases/novel-coronavirus-2019/technicalguidance.

3. Global Specialist Training Courses - https://openwho.org/ courses ; COVID-19: Global Specialist Training Courses https://openwho.org/channels/covid-19..

4. Health Force: Education and Training - https://www.who.int/ hrh/education/en/..

5. Dr Tedros Adhanom Ghebreyesus, WHO Director-General, notes at May 8 2020, Twitter - https://twitter.com/hashtag/ COVID19?src $=$ hashtag_click..

\section{c) (i) (8)}

This work is licensed under a Creative Commons AttributionNon Commercial 4.0 International License. 